\title{
Interventions to improve vaccine acceptance among rheumatoid arthritis patients: a systematic review
}

\author{
Vincent Gosselin Boucher ${ }^{1,2} \cdot$ Ines Colmegna ${ }^{3} \cdot$ Claudia Gemme $^{1,2} \cdot$ Sara Labbe $^{1,2} \cdot$ Sandra Pelaez $^{4} \cdot$ Kim L. Lavoie $^{1,2}$
}

Received: 28 December 2018 / Accepted: 1 January 2019 / Published online: 15 January 2019

(C) The Author(s) 2019, corrected publication 2019

\begin{abstract}
Introduction/objective National guidelines emphasize the importance of annual immunization for patients living with rheumatoid arthritis (RA), but vaccination rates remain suboptimal in this population. Evaluating the efficacy of patient and/or providertargeted interventions to improve vaccination uptake among RA patients could inform practice.

Methods We conducted a systematic review (SR) to examine the efficacy of interventions (exposure) aiming to improve vaccination uptake in patients with RA (outcome). English and French language, peer-reviewed interventional studies to improve vaccination rates in RA patients published between 2009 and 2018 were included.

Results The search yielded a total of 450 records. Five articles met inclusion criteria. All interventions focused on changing provider behavior using some form of vaccination reminder as the primary intervention strategy, though only two studies reported provider prescribing behavior as an outcome (which was $4 \%$ and $58 \%$ ). Overall, studies varied greatly regarding intervention delivery mode (e.g., educational sessions, e-mail reminders, best practice alerts), and behavior change techniques used to encourage providers to prescribe vaccination (e.g., feedback and monitoring, shaping knowledge, self-regulation). For influenza, pneumococcal and herpes zoster, post-intervention (mean 12-16 months follow-up) vaccination rates increased by a mean of $16.6 \%( \pm 15.4 \%)$.

Conclusions Interventions to enhance vaccine uptake in RA focused almost exclusively on improving provider prescription of vaccines using reminder-type interventions. Although effective in improving vaccination rates, those studies used heterogeneous interventions and behavior change techniques. Few studies measured provider prescribing behavior as an outcome. Future studies targeting providers should measure relevant provided-related outcomes and their impact on patient outcomes, to determine overall efficacy.
\end{abstract}

Keywords Behavioral interventions $\cdot$ Rheumatoid arthritis $\cdot$ Systematic review $\cdot$ Vaccination

Rheumatoid arthritis (RA), the most common musculoskeletal inflammatory disorder worldwide [1], affects approximately $1 \%$ of the population [2]. RA is a chronic systemic

Kim L. Lavoie

kiml_lavoie@yahoo.ca

1 Department of Psychology, University of Quebec at Montreal (UQAM), CP 8888, Sucursale Centre-Ville, Montreal, Quebec H3C 3P8, Canada

2 Montreal Behavioural Medicine Centre, Centre Intégré Universitaire de santé et services sociaux du Nord-de-l'Ile-de-Montréal (CIUSSS-NIM), Hôpital du Sacré-Coeur de Montréal Canada, Montréae, Canada

3 Department of Medicine, Division of Rheumatology, McGill University, Montreal, Canada

4 Department of Educational and Counselling Psychology, McGill University, Montreal, Canada autoimmune inflammatory disease that primarily manifests with synovitis, usually polyarticular. This disease is more common among women $(\mathrm{F} / \mathrm{M}$ ratio $=2: 1)$, and the lifetime risk of developing RA in adulthood is 3.6\% for women and $1.7 \%$ for men [3]. From a public health perspective, the costs associated with RA are substantial and estimated at $>\$ 39.2$ billion annually in the USA [4].

A substantial burden of RA relates to the increased morbidity and mortality associated with infectious diseases [5]. RA patients have higher risk of two major vaccinepreventable respiratory organisms: influenza and pneumococcus [6]. Further, people living with RA are two times more likely to develop medical complications that frequently require hospitalization due to those respiratory diseases than age-matched healthy controls $[7,8]$. This highlights the need to target RA patients for vaccination [5]. Despite current recommendations that identify RA patients as a high-priority group for vaccination [9], vaccination coverage among RA 
patients is suboptimal [10]. Reported immunization rates range between $25-90 \%$ for influenza and $17-62 \%$ for pneumococcus [10-15]. This is often below the target proposed by the World Health Assembly for seasonal flu vaccination coverage in at-risk populations which is $75 \%$ [16].

Improving vaccination rates is therefore a public health priority as it may enhance protection in adults living with RA as well as the community at large. Several factors have been associated with low vaccination rates in RA. Patient-related factors include high perceived vaccine risk and low perceived efficacy [17]. Provider-related factors include the failure to advocate for and prescribe vaccines to RA patients [14]. Although this topic is of high interest from a public health perspective, to our knowledge, no study has systematically reviewed the evidence on the nature and efficacy of interventions aiming to improve vaccination uptake among RA patients. Thus, the purpose of this review was to fill this knowledge gap by assessing the efficacy of existing interventions targeting either patients and/or health care providers aiming to improve vaccine uptake among RA patients. Since vaccination acceptance is a health-related behavior, we were particularly interested in behavioral interventions addressing vaccine uptake. Also, we aim to dissect the specific components (e.g., content, format, structure) of the most efficacious interventions.

\section{Methods}

The PRISMA checklist was followed to ensure transparent and comprehensive reporting throughout the systematic review [18]. The review was registered with the International Prospective Register of Systematic Reviews (PROSPERO: CRD42018103564) [19].

\section{Inclusion and exclusion criteria}

Studies reporting the results of interventions to improve vaccination uptake in RA were included. More specifically, studies on behavioral interventions [20] targeting providers and/or patients to enhance vaccination uptake among RA patients. There was no restriction on vaccine type (e.g., influenza, pneumococcal, herpes zoster). Only English or French publications in peer-reviewed journals reporting pre- and post-intervention measures of vaccination (e.g., vaccination rates) were included. Studies assessing interventions to improve vaccination rates in the general population or in chronic diseases other than RA (e.g., cancer, pulmonary disease) were excluded.

\section{Search strategy and review process}

PUBMED, PsychINFO, SCOPUS, and Cochrane searches up to July 25th 2018 were conducted. The keyword terms used were: "vaccination" AND "rheumatoid arthritis" AND "behaviour change", "behavior change", "motivational interviewing", "motivation communication", "counseling", "counselling", "behavioral", "intervention".

Reference lists of selected publications were screened to identify additional studies (see Table 1). This search process generated 450 unique and potentially eligible studies. As seen in Fig. 1, only five articles fulfilled the inclusion/exclusion criteria [21-25]. The following information was extracted from those five studies: participants (number and type of provider and RA patients included); outcomes of interest (e.g., vaccination rates, provider behaviors around vaccination prescribing); intervention characteristics (e.g., type, timing, structure, components of the intervention, follow-up period); comparison group characteristics (when applicable)

\section{Study quality}

Study quality was assessed by two independent evaluators using the Downs and Black checklist, a 27-item rating system assessing the methodological quality of randomized and nonrandomized interventions [26]. This checklist helps reviewers identify the strengths and weaknesses of the methodological design and reporting quality (e.g., randomization, blinding, reporting bias) of each reviewed study. Inter-rater agreements and kappa coefficients [27] were calculated with a 95\% confidence interval using the two raters' scores (V.G.B. and S.L.) for each study included in this review. This was done at the data extraction phase $(86 \%$, kappa $=0.7)$ and after study inclusion (study quality, 89\%, kappa $=0.7$ ). The inter-rater agreement score for the first round of eligibility assessment by titles and abstracts screening was also very high (90\%).

\section{Results}

\section{Study characteristics}

Study characteristics are summarized in Table 1. Out of five studies, three targeted providers and two providers and patients. The number of providers participating in the studies was generally small (8-35) or was not reported $(n=3$ studies) $[21,24,25]$. Patients in the studies ranged from 197 to 3717. The identified objective of the intervention was "improving vaccination rates" (in patients) [21-24], "reducing the frequency of any missed opportunities for vaccination" (in providers) [25], and "increase the documentation of prescriptions (in providers)" $[21,24]$. Three studies reported follow-up periods [22, 23, 25]. Time to post-intervention evaluations was generally 1 year, with two studies assessing patient vaccination rates monthly, for a median follow-up period of 12 [23] to 16 months [22]. 


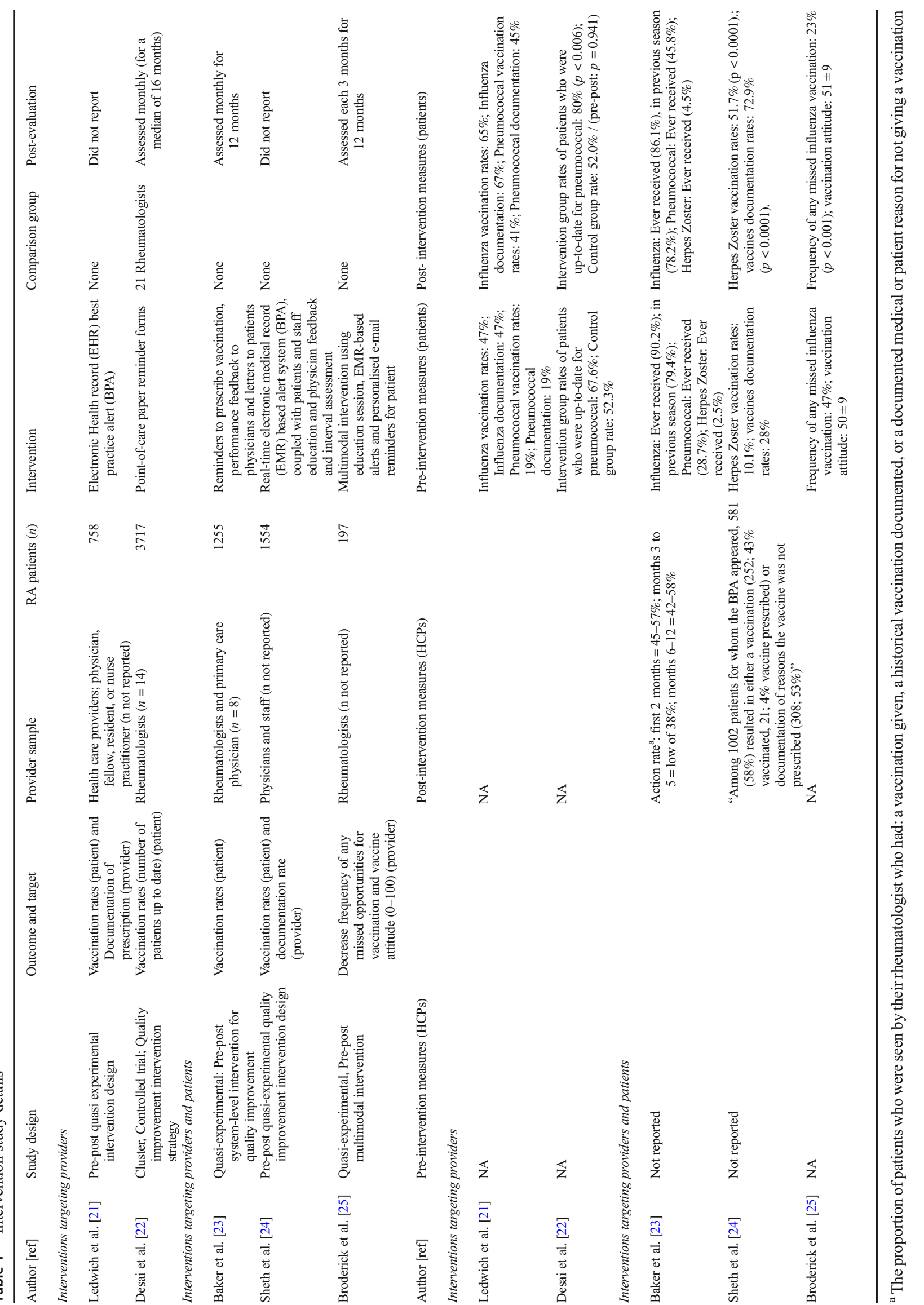




\section{Interventions' characteristics}

Intervention target, duration, content, and delivery mode varied considerably. All studies included interventions that targeted provider behavior to increase vaccination rates, and all provider interventions involved some form of vaccination reminder to discuss or prescribe vaccination as the primary intervention, including Electronic Health Record (EHR) or Electronic Medical Record (EMR) alerts and Point-of-care paper reminder forms. Only three studies also targeted patients and included education [24] or letters/e-mail reminders [23, 25] to improve vaccination rates. Intervention components varied considerably between studies. For this review, we defined an intervention component as any intervention strategy, implicitly or explicitly stated by the original study, as summarized by Michie's Taxonomy [20]. To achieve an adequate comparison of each intervention's content, the first (V.G.B.) and second (S.L.) reviewers identified their unique components and linked them to the Behavior Change Techniques (BCT's) identified by Michie and colleagues [20]. Among provider-targeted interventions, we identified a total of three BCTs used across the two studies and included: (1) adding objects to the environment (i.e., paper reminder forms, $1 / 2$ studies) [22], (2) monitoring behavioral outcomes (EHR best practice alerts, 1/2 studies) [21], and (3) pharmacological support (provided vaccination documentation or advice to get vaccinated, $2 / 2$ studies) $[21,22]$. Among patienttargeted interventions, we identified a total of five BCTs including (1) adding objects to the environment (i.e., paper reminder forms, 2/3 studies) [23, 25], (2) providing feedback on behavior (i.e., performance feedback, $2 /$ 3 studies) [23, 24], (3) monitoring behavioral outcomes (EMR-based alert, 2/3 studies) [24, 25], (4) instruction on how to perform the behavior (educational session (s) with follow-up assessments; $2 / 3$ studies) [24, 25], and (5) pharmacological support (provided vaccination documentation or advice to get vaccinated, 3/3 studies) [23-25] (see Table 2).

Few studies provided information on the structure of the intervention. Only two studies provided formal education sessions $[24,25]$, the content of which varied: Broderick et al. focused on the rationale, efficacy, and recommended uses of vaccination [25], while Sheth and colleagues [24] had providers engage in small group discussions to address concerns, clarify misconceptions, and update recommendations regarding vaccination [24]. The interventions were brief at only one [24] or two [25] sessions. None of these studies reported the duration (e.g., total minutes/hours/days) of the sessions, trainers' qualifications, trainee attendance or participation, or training program fidelity.

\section{Interventions' results}

Due to the high heterogeneity of intervention components and the lack of standardized reporting of outcomes, we were unable to perform a formal meta-analysis. However, the interventions included in this review were shown to be effective (see Table 1). For the three types of vaccines targeted (influenza, pneumococcal, and herpes zoster), pre-intervention vaccination rates ranged from 47 to $79.4 \%$ for influenza vaccine [21, 23], 19 to $28.7 \%$ for pneumococcal vaccine [21, 23], and 2.5 to $10.1 \%$ for herpes zoster vaccine $[21,23]$. Post-intervention (12-month follow-up) vaccination rates increased by a mean of $8.4 \pm 13.6 \%$ for influenza, $19.6 \pm 3.5 \%$ for pneumococcal, and $21.8 \pm 28.0 \%$ for herpes zoster vaccine. These were statistically significant improvements. Broderick and colleagues measured the frequency of any missed influenza vaccination pre-intervention $(47 \%)$ and post-intervention $(23 \% ; p<0.01)$. In the only controlled study with a comparison group, rheumatologist did not receive paper reminder [22], pre-intervention rates of pneumococcal vaccination were $67.6 \%$ in the intervention group and $52.3 \%$ in the control group. Post-intervention (median of 16-month follow-up) pneumococcal vaccination rates were $80 \%(+12.3, p<0.01)$ in the intervention group and $52 \%(-0.3, p=0.09)$ in the comparison group.

Although all studies targeted providers, only two reported post-intervention measures of prescription behaviors [23, 24] and none baseline frequencies. Baker and colleagues [23] reported the "action rate" of providers (the proportion of patients who were seen by their rheumatologist who had a vaccination given, a historical vaccination documented, or a documented medical or patient reason for not giving a vaccination), which varied from 38 to $58 \%$ during the 12 months of implementation. The second study reported the proportion of patients that were vaccinated $(43 \%)$ or for whom a vaccine was ordered (4\%) or for whom a reason for not getting vaccinated was documented (3\%: physician deferred [27\%] or patients declined [73\%]). Also, two studies reported pre-post vaccination prescription documentation rates with a mean increase of $30 \%$ [21, 24]. Finally, one study evaluated vaccination attitudes among RA patients (the Vaccine Attitudes Questionnaires, score 0-100) [25] and showed no change from pre-post intervention ( $50 \pm 9$ to $51 \pm 9 ; p=0.58$ ).

\section{Study quality}

The methodological quality of the studies varied considerably (Downs and Black [26] checklist score range: 11-15) with an average score of 13 out of 28 denoting moderate quality. None of the studies received an excellent rating (26-27) or scores below 10 that are considered of poor quality (see appendix for scores). The low-quality scores of the studies were mostly attributable to non-randomized designs and the lack of comparison groups. 
Fig. 1 Flow diagram

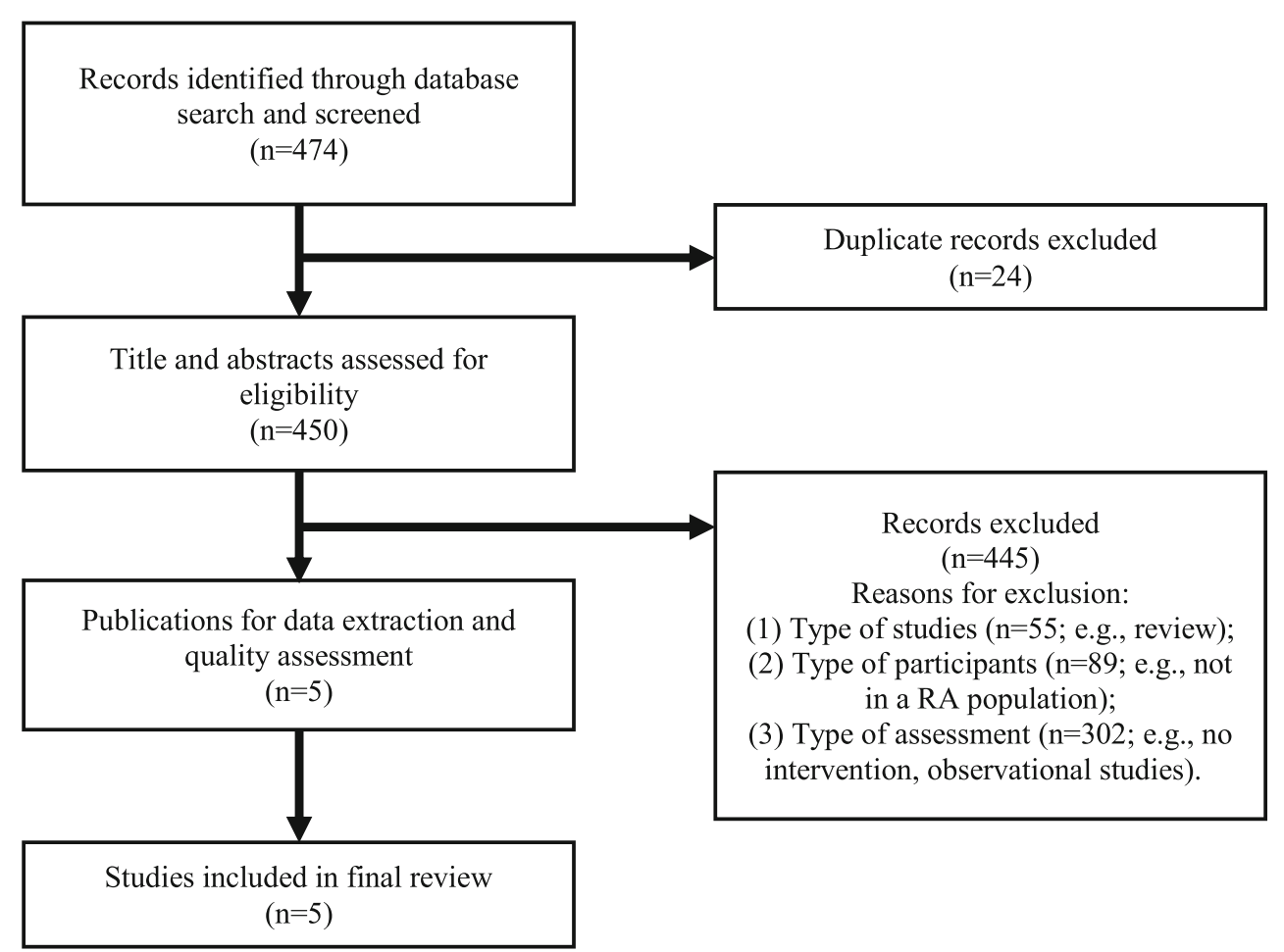

\section{Discussion}

This study reviewed the existing literature addressing the impact of behavioral interventions on vaccine uptake among RA patients. Overall, few studies have been conducted to date targeting this topic in this population. Reminder-type interventions were the most commonly used interventions to improve provider prescription of vaccines and vaccine uptake among RA patients. Despite the narrow focus on primarily provider-targeted interventions involving reminders to vaccinate, intervention strategies (e.g., Electronic Medical Record [EMR] alerts, point-of-care paper and electronic reminders, practice feedback) were heterogeneous, making impossible to conduct a formal meta-analysis to assess the overall magnitude of their effects. Although all studies reported improvements in vaccination rates among RA patients, none actually measured provider prescription behavior pre- and post-intervention, which makes it difficult to determine the true efficacy of the intervention. When conducting behavioral interventions, measuring behavioral mediators (e.g., vaccination prescription by providers),

Table 2 Intervention components

\begin{tabular}{|c|c|c|c|c|c|}
\hline Behavior Change Technique Taxonomy (Michie et al. 2013) & $\begin{array}{l}\text { Ledwich et al. } \\
\text { [21] }\end{array}$ & $\begin{array}{l}\text { Desai et al. } \\
{[22]}\end{array}$ & $\begin{array}{l}\text { Baker et al. } \\
{[23]}\end{array}$ & $\begin{array}{l}\text { Sheth et al. } \\
{[24]}\end{array}$ & $\begin{array}{l}\text { Broderick et al. } \\
{[25]}\end{array}$ \\
\hline \multicolumn{6}{|l|}{ Interventions targeting providers only } \\
\hline $\begin{array}{l}\text { Feedback and monitoring (2.) Monitoring of outcomes behavior } \\
\text { without feedback (2.5) }\end{array}$ & $\sqrt{ }$ & & & & \\
\hline Regulation (11.) Pharmacological support (11.1) & $\sqrt{ }$ & $\sqrt{ }$ & & & \\
\hline Antecedents (12.) Adding objects to the environment (12.5) & & $\sqrt{ }$ & & & \\
\hline \multicolumn{6}{|l|}{ Interventions targeting providers and patients } \\
\hline Feedback and monitoring (2.) Feedback on behavior (2.2) & & & $\sqrt{ }$ & $\sqrt{ }$ & \\
\hline Monitoring of outcomes behavior without feedback (2.5) & & & & $\sqrt{ }$ & $\sqrt{ }$ \\
\hline $\begin{array}{l}\text { Shaping knowledge (4.) Instruction on how to perform the behavior } \\
\text { (4.1) }\end{array}$ & & & & $\sqrt{ }$ & $\sqrt{ }$ \\
\hline Regulation (11.) Pharmacological support (11.1) & & & $\sqrt{ }$ & $\sqrt{ }$ & $\sqrt{ }$ \\
\hline Antecedents (12.) Adding objects to the environment (12.5) & & & $\sqrt{ } \mathrm{a}^{2}$ & & $\sqrt{ } \mathrm{a}^{\mathrm{a}}$ \\
\hline
\end{tabular}

${ }^{\mathrm{a}}$ Only the patients 
it is key to assess the interventions' hypothesized mechanism(s) of action on clinical outcomes [28] (e.g., vaccine uptake among patients). All studies reviewed focused on changing provider vaccination prescribing behavior as the primary means of increasing vaccination rates, but all failed to systematically assess pre- and post-intervention rates of vaccination prescription. Investigators in this area are encouraged to measure changes in these intermediate behavioral targets, as well as the association between these changes and clinical outcomes, in order to determine the true effectiveness of these interventions. Finally, generalizability of the reminder-type interventions is limited by the poor methodological reporting of intervention details including intervention schedule (number of sessions), dose (duration of intervention), or educator details. Failure to report this information does not allow study replication, limiting their value and potential impact.

Only three studies included interventions that also targeted patients. However, the failure to measure provider prescription behavior makes it impossible to know which intervention targets were more effective: those targeting the providers or those targeting providers and patients, as only patient-level outcomes (vaccination rates) were measured.

In general, the results of this review are consistent with similar studies using reminder-based interventions targeting physicians to improve vaccination rates in the context of vaccination $[29,30]$. However, they were not consistent (no significant change post-intervention) with one study that provided letters to physicians with the aim of reducing missing vaccination opportunities to improve MMR vaccination among children [31]. It is possible that when vaccines target children, where parents are making decisions on their behalf, provider reminders that do not address the complex concerns of parents are not as effective. Additionally, the results of the present review were also consistent with studies using reminderbased interventions targeting patients with other diseases. For example, two studies using brochure reminders [32] or mailed reminders [33] showed significant increases in vaccination rates post-intervention among patients at high risk of infections (ranging from 1.6 to $6 \%$ higher rates). However, not all studies using reminder-based interventions with patients at risk of infection were effective [34, 35]. Finally, two studies failed to observe increases in MMR immunization rates in association with text messages targeting pregnant women [35] and telephone reminders with home visits among parents [34]. Once again, it is possible that reminder-based interventions are insufficient to address the complex concerns of parents around immunization of their children.

\section{Limitations}

This review is limited by the low number of studies meeting inclusion criteria, the variety of their interventions and the methodological heterogeneity that precludes conducting a formal meta-analysis, and the inclusion of studies of generally moderate methodological quality. Key limitations of the studies included in this review include the lack of randomized designs and absence of comparison groups, low sample sizes, inadequate reporting of methodological details (duration, dose), and the failure to measure targeted behavioral outcomes (vaccination prescription) among providers. An additional missing component of all studies was the lack of reported stakeholder involvement in intervention protocol development. The integration of stakeholders (e.g., patients, HCPs, administrators) is encouraged to identify research priorities, define relevant outcomes, and help clinical translation/implementation [36, 37].

\section{Conclusion}

This review highlights the paucity of research on the efficacy of interventions designed to improve vaccination uptake among RA patients, despite the sub-optimal vaccination rates and the fact that RA patients are a high-risk population. Furthermore, this review indicates that all interventions to date have focused on changing provider behavior to improve vaccination rates, without interventions targeting specifically patients' factors such as perceived lack of vaccine efficacy or concerns over side effects. Accepting to be vaccinated is a complex behavior that relies upon both provider and patient factors that will likely be inadequately addressed in interventions focusing exclusively on one or the other. Consequently, future studies should develop and test interventions targeting both provider and patient behavior. One such intervention may be motivational communication (MC), which help providers educate, motivate, and enable patients to engage in appropriate and beneficial self-management behaviors to improve chronic disease outcomes [38,39]. This approach involves training providers in evidence-based behavior change techniques that focus on shared-decision-making that links patients' health objectives (e.g., higher number of pain-free days, improved mobility) to engaging in positive health behaviors (e.g., vaccination). Interventions using MC-based strategies have been shown to be associated with improvements in a wide range of health behaviors (e.g., medication adherence, physical activity, and exercise) and clinical outcomes (e.g., patient health). The extent to which MC may be efficacious for improving vaccination rates among RA patients remains to be determined, but could be promising to address this complex behavior.

Acknowledgements We acknowledge investigator support from the Fonds de la recherche du Quebec-Santé (FRQS, KLL), scholarship support from the Canadian Institutes of Health Research (CIHR, VGB), the FRQS (VGB, SL), and the J.A. De Seve Foundation (CG). 
Funding information This work was funded by an operating grant from the Canadian Initiative for Outcomes in Rheumatology cAre (CIORA), Canadian Rheumatology Association (Dr Colmegna and Dr. Lavoie are co-PIs).

\section{Compliance with ethical standards}

\section{Disclosures None.}

Open Access This article is distributed under the terms of the Creative Commons Attribution 4.0 International License (http:// creativecommons.org/licenses/by/4.0/), which permits unrestricted use, distribution, and reproduction in any medium, provided you give appropriate credit to the original author(s) and the source, provide a link to the Creative Commons license, and indicate if changes were made.

Publisher's note Springer Nature remains neutral with regard to jurisdictional claims in published maps and institutional affiliations.

\section{References}

1. Cooper GS, Bynum ML, Somers EC (2009) Recent insights in the epidemiology of autoimmune diseases: improved prevalence estimates and understanding of clustering of diseases. J Autoimmun 33(3-4):197-207

2. World Health Organization (2018) Chronic disease and health promotion. Retrieved from https://www.who.int/chp/topics/rheumatic/en/. Accessed 2 Dec 2018

3. Crowson CS, Matteson EL, Myasoedova E, Michet CJ, Ernste FC, Warrington KJ, Davis JM III, Hunder GG, Therneau TM, Gabriel SE (2011) The lifetime risk of adult-onset rheumatoid arthritis and other inflammatory autoimmune rheumatic diseases. Arthritis Rheum 63(3):633-639. https://doi.org/10.1002/art.30155

4. Birnbaum H, Pike C, Kaufman R, Maynchenko M, Kidolezi Y, Cifaldi M (2010) Societal cost of rheumatoid arthritis patients in the US. Curr Med Res Opin 26(1):77-90. https://doi.org/10.1185/ 03007990903422307

5. Perry LM, Winthrop KL, Curtis JR (2014) Vaccinations for rheumatoid arthritis. Curr Rheumatol Rep 16(8):431. https://doi.org/10. 1007/s11926-014-0431-x

6. Blumentals WA, Arreglado A, Napalkov P, Toovey S (2012) Rheumatoid arthritis and the incidence of influenza and influenzarelated complications: a retrospective cohort study. BMC Musculoskelet Disord 13(158). https://doi.org/10.1186/14712474-13-158

7. Doran MF, Crowson CS, Pond GR, O'Fallon WM, Gabriel SE (2002) Frequency of infection in patients with rheumatoid arthritis compared with controls: a population-based study. Arthritis Rheum 46(9):2287-2293. https://doi.org/10.1002/art.10524

8. Meroni PL, Zavaglia D, Girmenia C (2018) Vaccinations in adults with rheumatoid arthritis in an era of new disease-modifying antirheumatic drugs. Clin Exp Rheumatol 36(2):317-328

9. Bombardier C, Hazlewood GS, Akhavan P, Schieir O, Dooley A, Haraoui B, Khraishi M, Leclercq SA, Légaré J, Mosher DP, Pencharz J, Pope JE, Thomson J, Thorne C, Zummer M, Gardam MA, Askling J, Bykerk V, Canadian Rheumatology Association (2012) Canadian rheumatology association recommendations for the pharmacological management of rheumatoid arthritis with traditional and biologic disease-modifying antirheumatic drugs: part II safety. J Rheumatol 39(8):1583-1602. https://doi.org/10.3899/ jrheum.120165
10. Hmamouchi I, Winthrop K, Launay O, Dougados M (2015) Low rate of influenza and pneumococcal vaccine coverage in rheumatoid arthritis: data from the international COMORA cohort. Vaccine 33(12):1446-1452. https://doi.org/10.1016/j.vaccine.2015.01.065

11. Brocq O, Acquacalda E, Berthier F, Albert C, Bolla G, Millasseau E, Destombe C, Azulay J, Asquier C, Florent A, le Seaux S, EullerZiegler L (2016) Influenza and pneumococcal vaccine coverage in 584 patients taking biological therapy for chronic inflammatory joint: a retrospective study. Joint Bone Spine 83(2):155-159. https://doi.org/10.1016/j.jbspin.2015.11.005

12. Caldera F, Saha S, Wald A, Cooley DM, Zhao YQ, Li Z, Bartels CM (2016) Comparing guideline-based care quality for inflammatory bowel disease and rheumatoid arthritis patients within a medical home. Expert Rev Gastroenterol Hepatol 10(6):759-766. https://doi.org/10.1586/17474124.2016.1169920

13. Costello R, Winthrop KL, Pye SR, Brown B, Dixon WG (2016) Influenza and pneumococcal vaccination uptake in patients with rheumatoid arthritis treated with immunosuppressive therapy in the UK: a retrospective cohort study using data from the clinical practice research datalink. PLoS One 11(4):e0153848. https://doi. org/10.1371/journal.pone. 0153848

14. Hua C, Morel J, Ardouin E, Ricard E, Foret J, Mathieu S, Combe B, Lukas C (2015) Reasons for non-vaccination in French rheumatoid arthritis and spondyloarthritis patients. Rheumatology 54(4):748750. https://doi.org/10.1093/rheumatology/keu531

15. Krasselt M, Ivanov JP, Baerwald C, Seifert O (2017) Low vaccination rates among patients with rheumatoid arthritis in a German outpatient clinic. Rheumatol Int 37(2):229-237. https://doi.org/10. 1007/s00296-016-3608-y

16. Blank P, Schwenkglenks M, Szucs TD (2012) The impact of European vaccination policies on seasonal influenza vaccination coverage rates in the elderly. Hum Vaccin Immunother 8(3):328 335

17. Williams WW, Lu PJ, O'Halloran A et al (2017) Surveillance of vaccination coverage among adult populations - United States, 2015. MMWR Surveill Summ 66(11):1-28. https://doi.org/10. 15585/mmwr.ss6611a1

18. Liberati A, Altman DG, Tetzlaff J, Mulrow C, Gotzsche PC, Ioannidis JPA, Clarke M, Devereaux PJ, Kleijnen J, Moher D (2009) The PRISMA statement for reporting systematic reviews and meta-analyses of studies that evaluate healthcare interventions: explanation and elaboration. BMJ 339:b2700. https://doi.org/10. 1136/bmj.b2700

19. Boucher VG, Colmegna I, Gemme C, Dragomir AI, Labbé S, Pélaez S, Lavoie KL (2018) Interventions to improve vaccine acceptance among rheumatoid arthritis patients: a systematic review. PROSPERO 2018 CRD42018103564 Available from: www.crd. york.ac.uk/PROSPERO/display_record.php?ID= CRD42018103564

20. Michie S, Richardson M, Johnston M (2013) The behavior change technique taxonomy (v1) of 93 hierarchically clustered techniques: building an international consensus for the reporting of behavior change interventions. Ann Behav Med 46(1):81-95. https://doi. org/10.1007/s12160-013-9486-6

21. Ledwich LJ, Harrington TM, Ayoub WT, Sartorius JA, Newman ED (2009) Improved influenza and pneumococcal vaccination in rheumatology patients taking immunosuppressants using an electronic health record best practice alert. Arthritis Rheum 61(11): 1505-1510. https://doi.org/10.1002/art.24873

22. Desai SP, Lu B, Szent-Gyorgyi LE, Bogdanova AA, Turchin A, Weinblatt M, Coblyn J, Greenberg JO, Kachalia A, Solomon DH (2013) Increasing pneumococcal vaccination for immunosuppressed patients: a cluster quality improvement trial. Arthritis Rheum 65(1):39-47. https://doi.org/10.1002/art.37716

23. Baker DW, Brown T, Lee JY, Ozanich A, Liss DT, Sandler DS, Ruderman EM (2016) A multifaceted intervention to improve 
influenza, pneumococcal, and herpes zoster vaccination among patients with rheumatoid arthritis. J Rheumatol 43(6):1030-1037. https://doi.org/10.3899/jrheum. 150984

24. Sheth H, Moreland L, Peterson H, Aggarwal R (2017) Improvement in herpes zoster vaccination in patients with rheumatoid arthritis: a quality improvement project. J Rheumatol 44(1): 11-17. https://doi.org/10.3899/jrheum.160179

25. Broderick R, Ventura I, Soroosh S, Franco L, Giles JT (2018) Reducing missed opportunities for influenza vaccination in patients with rheumatoid arthritis: evaluation of a multisystem intervention. $\mathrm{J}$ Rheumatol 45:1220-1228. https://doi.org/10.3899/jrheum.170763

26. Downs SH, Black N (1998) The feasibility of creating a checklist for the assessment of the methodological quality both of randomised and non-randomised studies of health care interventions. J Epidemiol Community Health 52(6):377-384

27. McHugh ML (2012) Interrater reliability: the kappa statistic. Biochemia Medica 22(3):276-282

28. Czajkowski SM, Powell LH, Adler N (2015) From ideas to efficacy: the ORBIT model for developing behavioral treatments for chronic diseases. Health Psychol 34(10):971-982. https://doi.org/ 10.1037/hea0000161

29. Chambers CV, Balaban DJ, Carlson BL, Grasberger DM (1991) The effect of microcomputer-generated reminders on influenza vaccination rates in a university-based family practice center. J Am Board Fam Pract 4(1):19-26

30. Chan L, MacLehose RF, Houck PM (2002) Impact of physician reminders on the use of influenza vaccinations: a randomized trial. Arch Phys Med Rehabil 83(3):371-375

31. Muehleisen B, Baer G, Schaad UB, Heininger U (2007) Assessment of immunization status in hospitalized children followed by counseling of parents and primary care physicians improves vaccination coverage: an interventional study. J Pediatr 151(6): 704-706
32. Thomas DM, Ray SM, Morton FJ, Drew J, Offutt G, Whitney CG, Jacobson TA (2003) Patient education strategies to improve pneumococcal vaccination rates: randomized trial. J Investig Med 51(3): $141-148$

33. Ahmed F, Friedman C, Franks A (2004) Effect of the frequency of delivery of reminders and an influenza tool kit on increasing influenza vaccination rates among adults with high-risk conditions. Am J Manag Care 10(10):698-702

34. Lemstra M, Rajakumar D, Thompson A, Moraros J (2011) The effectiveness of telephone reminders and home visits to improve measles, mumps and rubella immunization coverage rates in children. Paediatr Child Health 16(1):e1-e5

35. Moniz MH, Hasley S, Meyn LA, Beigi RH (2013) Improving influenza vaccination rates in pregnancy through text messaging: a randomized controlled trial. Obstet Gynecol 121(4): 734-740

36. McCarthy EA, Pollock WE, Nolan T, Hay S, McDonald S (2012) Improving influenza vaccination coverage in pregnancy in Melbourne 2010-2011. Aust N Z J Obstet Gynaecol 52(4):334-341

37. Sowden E, Mitchell WS (2007) An audit of influenza and pneumococcal vaccination in rheumatology outpatients. BMC Musculoskelet Disord 8(1):58

38. Lavoie KL, Moullec G, Lemiere C et al (2014) Efficacy of brief motivational interviewing to improve adherence to inhaled corticosteroids among adult asthmatics: results from a randomized controlled pilot feasibility trial. Patient Prefer Adherence 8:1555-1569. https://doi.org/10.2147/ppa.s66966

39. Rouleau CR, Lavoie KL, Bacon SL, Vallis M, Corace K, Campbell TS (2015) Training healthcare providers in motivational communication for promoting physical activity and exercise in Cardiometabolic health settings: do we know what we are doing? Curr Cardiovasc Risk Rep 9(6):29. https://doi.org/10.1007/s12170015-0457-2 